\title{
Incidence of thromboembolism in patients with COVID-19: a systematic review and meta-analysis
}

\author{
Kochawan Boonyawat ${ }^{1 *}$ D, Pichika Chantrathammachart ${ }^{1}$, Pawin Numthavaj ${ }^{2}$, Nithita Nanthatanti ${ }^{3}$, \\ Sithakom Phusanti ${ }^{3}$, Angsana Phuphuakrat ${ }^{1}$, Pimjai Niparuck ${ }^{1}$ and Pantep Angchaisuksiri ${ }^{1}$
}

\begin{abstract}
Background: Since the beginning of the coronavirus disease 2019 (COVID-19) pandemic, the incidence of thromboembolism has been increasingly reported. The aim of this systematic review was to explore the incidence of venous and arterial thromboembolism among COVID-19 patients requiring hospitalization.

Methods: Medline, Embase, Scopus, and grey literature were searched until June 2020. Observational studies reported on the incidence of venous thromboembolism (VTE), including pulmonary embolism (PE) and deep vein thrombosis (DVT) or arterial thromboembolism (ATE) were included. The pool incidences and their 95\% confidence intervals $(\mathrm{Cl})$ were calculated using the random-effects model.

Results: A total of 36 studies were included. In the intensive care unit (ICU) setting, the pooled incidence of VTE was $28 \%$ (95\% Cl, 22-34\%). Subgroups based on compression ultrasound (CUS) screening revealed a higher incidence of DVT in the CUS screening group than in the no CUS screening group (32\% [95\% Cl, 18-45\%] vs. 6\% [95\% Cl, 4-9\%]). The pooled incidence of ATE in ICU was 3\% (95\% Cl, 2-5\%). In the non-ICU setting, the pooled incidence of VTE was 10\% (95\% Cl, 6-14\%).

Conclusions: The incidence of VTE in COVID-19 patients was higher in the ICU setting than in the non-ICU setting, and also significantly higher in studies that incorporated the CUS screening protocol. The incidence of ATE in the ICU setting was low. VTE prophylactic measures should be given to all hospitalized patients diagnosed with COVID-19.
\end{abstract}

Keywords: COVID-19, Venous thromboembolism, Arterial thromboembolism, Meta-analysis

\section{Background}

Since December 2019, coronavirus disease 2019 (COVID-19) has emerged as a pandemic, causing high morbidity and mortality. The association between coagulation abnormalities, including disseminated intravascular coagulation and hypercoagulable state, and COVID-19 has been increasingly reported. The proposed underlying mechanism is that coronavirus infection could

\footnotetext{
* Correspondence: Kochawan.boo@mahidol.ac.th

'Department of Medicine, Faculty of Medicine Ramathibodi Hospital, Mahidol University, Bangkok, Thailand

Full list of author information is available at the end of the article
}

activate multiple systemic coagulation and inflammatory responses. Host inflammatory responses result in increased proinflammatory cytokine production, which leads to activation of coagulation and consumptive coagulopathy [1]. Several observational studies demonstrated a higher incidence of venous thrombotic events in patients diagnosed with COVID-19 admitted to the intensive care unit (ICU) compared with those from historical data [2, 3]. For arterial thrombosis, sepsisinduced coagulopathy with vascular endothelial dysfunction could contribute to microcirculatory changes in those diagnosed with COVID-19. However, few studies have 
reported on arterial thrombotic events in patients with COVID-19 [4]. In addition, most studies were case series and case report, which preclude the estimate of the incidence [5-7]. Anticoagulant prophylaxis is recommended by expert consensus for all critically ill COVID-19 patients, although breakthrough venous thrombosis was reported [8-10].

To date, no pre-existing systematic review and meta-analysis has addressed this issue. We conducted this systematic review to demonstrate the pooled incidence of venous and arterial thromboembolism in COVID-19 patients in various settings. The protocol was registered in PROSPERO (ID CRD42020182981).

\section{Methods}

Data source and literature search

A literature search was performed through bibliographic databases, including MEDLINE/Pubmed (1946 to present) using the OVID platform, Embase, and Scopus. Grey literature was searched through Google scholar and preprint servers, including MedRxiv and SSRN. For MEDL INE and Embase, search terms were available in the supplementary index. Lists of references of relevant articles and reviews were manually reviewed and screened for potential eligibility. There was no language restriction and no filtered used for study design. Studies with languages other than English were translated using the Google Translate tool. The search was performed on May 7th, 2020. We updated the search in grey literature on June 30th, 2020.

\section{Study selection}

Two researchers (K.B and P.C) independently screened titles and abstracts of the retrieved studies using inclusion and exclusion criteria. Review articles and references were searched for the possible included studies. Studies met eligibility criteria were included. Studies were eligible if the study design was an observational study that reported the incidence or prevalence of venous or arterial thrombosis in patients with confirmed COVID-19 requiring hospitalization. Studies with data available for incidence calculation were also included. Studies that were case-series that did not have data available for incidence calculation were excluded. Studies that were case-report, review, comments, consensus, or guidance in design were excluded. The outcome of venous thromboembolism (VTE) included pulmonary embolism (PE) and deep vein thrombosis (DVT) which were proximal, distal, and catheter-related DVT. The outcome of arterial thrombosis included ischemic stroke, myocardial infarction, and limb ischemia.

Full-text eligibility was assessed by two independent researchers (K.B and P.C). The disagreement was solved through discussion between the two researchers. If the disagreement persisted, the decision was made by the third adjudicator (A.P).

\section{Data abstraction and quality assessment}

Two independent reviewers (K.B, P.N) independently abstracted the data. Data of study (year, author, study design), patients characteristics (age, sex, comorbidity), the severity of the disease, patient's settings including ICU or non-ICU settings, number of events in each type of thrombosis, location of $\mathrm{PE}$, number of patients requiring hospitalization, ICU admission, or non-ICU admission were recorded. If available, the number of fatal PE, thromboembolic-related mortality, and all-cause mortality were also recorded. Upon data abstraction, we found variable inclusion criteria in each study. We have categorized the included studies in clinical and imaging studies. Clinical studies were studies that recorded the incidence of thrombotic events based on clinical data. Imaging studies were studies that recorded the incidence of thrombotic events based on imaging.

The risk of bias was assessed by two independent researchers (K.B, P.N). The disagreement was solved through discussion between two researchers. Regarding no standard of risk of the bias assessment tool [11], we used the risk of bias in the prevalence study proposed by Hoy et al. [12], which comprised four domains for external validity and six domains for internal validity. Criteria for external validity comprised representation of the target population, random selection, minimization of non-response bias. Criteria for internal validity comprised data collection, acceptable definition of the outcome, reliability, and validity in measurement tool, length of follow-up, and the correction of incidence report. We acknowledged that this assessment tool was aimed for a population-based prevalence study. Thus some criteria might not be applicable to our included studies.

\section{Statistical analysis}

The baseline characteristics of each study were summarized. Pooled incidences and respective confidence intervals (CI) of venous and arterial thrombosis were calculated from proportions extracted from reported studies. Clinical study and imaging study were analyzed separately. Meta-analysis was performed using an exact binomial random-effects model with inverse variance weighting method as we expected high heterogeneity between patients' populations, various diagnostic utilities, and preventive strategies. Continuity correction were applied on calculations made on studies which reported zero patients. We prespecified subgroup analyses based on clinical severity, eastern and western countries, and the use of anticoagulant prophylaxis. However, most clinical studies reported outcomes only in the ICU setting with only few reported outcomes in both ICU and/ 
or non-ICU settings. Therefore, we did not perform a subgroup analysis based on clinical severity. Furthermore, we found that some studies had a protocol for leg compression ultrasound (CUS) screening. We added a post hoc subgroup analysis of studies with leg CUS screening since these could lead to increased DVT incidence. We also performed and reported subgroup analysis based on country of studies to illustrate the differences in the incidence of VTE. Heterogeneity was explored using the Cochrane Q test. A $p$-value of $<0.05$ was considered statistically significant. $\mathrm{I}^{2}$ statistic was calculated to estimate heterogeneity. All statistical analyses and meta-analyses were performed using the metaprop command [13] with Stata software version 16 (Stata, College Station, TX, USA).

\section{Results}

Search results and flow of search strategies are available in the supplementary index. We identified a total of 762 articles (395 articles from Medline, 320 articles from Embase, 47 from Scopus). Grey literature search revealed 1900 articles from Google scholar, MedRxiv, and SSRN. After duplicates were removed, there were 1126 articles among which we underwent title and abstract screening. Of these, 935 articles were excluded. The remaining 191 full-text articles were assessed for eligibility criteria, and 170 articles were excluded (Supplementary index). The most common reasons for exclusion were case report or case series, which did not provide data or incidence reports and review articles. A total of 22 studies were included in the analysis. We performed a grey literature search on June 30th, 2020, and found 14 articles relevant and met the eligibility criteria.

\section{Risk of bias assessment}

All 36 studies were assessed for risk of bias using risk of bias for prevalence studies [12]. Most studies were subjected to moderate risk of bias owing to the representative of the population since they specifically reported outcomes in ICU or non-ICU settings for which were not representative of all hospitalization with COVID-19. All but seven studies were retrospective in design. All imaging studies have a high risk of bias due to selective patients who underwent imaging studies. In addition, the indications for computed tomography pulmonary angiography (CTPA) or CUS were varied between studies. We found that the criterion on national representative of the population was not applicable to the included studies since they were not population-based prevalence studies. The internal validity criterion of prevalence period was also not applicable due to, in our study, we intended to assess the prevalence of symptoms/complications (thrombotic outcomes) rather than the prevalence of the actual disease. The risk of bias assessment is presented in supplementary index.

\section{Characteristics of the included studies}

A total of 36 studies were included. Characteristics of the included studies are shown in Tables 1 and 2. Twentynine studies were retrospective, and 7 studies were prospective studies. Twenty-seven studies were from Europe (9 from France, 6 from Italy, 3 from the Netherland, 4 from Spain, 2 from the United Kingdom (UK), one each from Belgium, Germany and Switzerland). Three studies were from the United States of America (USA). Six studies were from China. There were 28 clinical and 8 imaging studies. The diagnosis of COVID-19 in most studies required the detection of severe acute respiratory syndrome coronavirus 2 (SARS-CoV-2) by real-time polymerase chain reaction (RT-PCR), but some were based on high clinical suspicion without the PCR results. In the clinical studies, 16 and 5 studies reported the incidences of outcomes only in the ICU $[2,3,8,14-26]$ or non-ICU settings [27-31], respectively. Seven studies reported the incidence of outcomes in the ICU, and non-ICU settings $[4,9,10,32-35]$. Six studies reported on both venous and arterial thrombosis $[2,14,16,18,21,33]$. Twenty-nine studies reported only venous thrombotic events, and one study reported only arterial thrombotic events [4]. Among 23 in 29 studies reported venous thrombotic outcomes, utilized anticoagulant prophylaxis. In the imaging studies, 6 studies mainly focused on CTPA in all hospitalization patients [36-41], and 2 studies focused on using compression ultrasound (CUS) in a non-ICU setting $[42,43]$.

\section{Clinical study}

VTE in ICU setting A total of 21 clinical studies were included. VTE occurred in 465 of 1766 patients with COVID-19 admitted in the ICU. The pooled incidence of total VTE, including DVT and PE was 28\% (95\% CI, $22-34 \%, \mathrm{I}^{2}=89.5$ ) (Fig. 1). The pooled incidence of PE was $3 \%\left(95 \% \mathrm{CI}, 2-4 \%, \mathrm{I}^{2}=94.3\right)$. The pooled incidence of DVT was $15 \%$ (95\% CI, 11-20\%, I ${ }^{2}=92.6$ ) (Figures S1 and S2 in the supplementary index).

Subgroup analyses of VTE based on anticoagulant prophylaxis, eastern or western countries, and CUS screening did not reveal significant differences between subgroups (Figures S3-S5 in the supplementary index). When focusing on the incidence of DVT, subgroup analysis based on CUS screening demonstrated a significant interaction $(p<0.001)$. In 12 studies with no CUS screening, the incidence of DVT was 6\% (95\% CI, 4 9\%), whereas in 9 studies with CUS screening, the incidence of DVT was 32\% (95\% CI, 18-45\%) (Fig. 2).

Overall, studies from the Netherlands, France, China, Italy and UK demonstrated the pooled VTE incidence of 


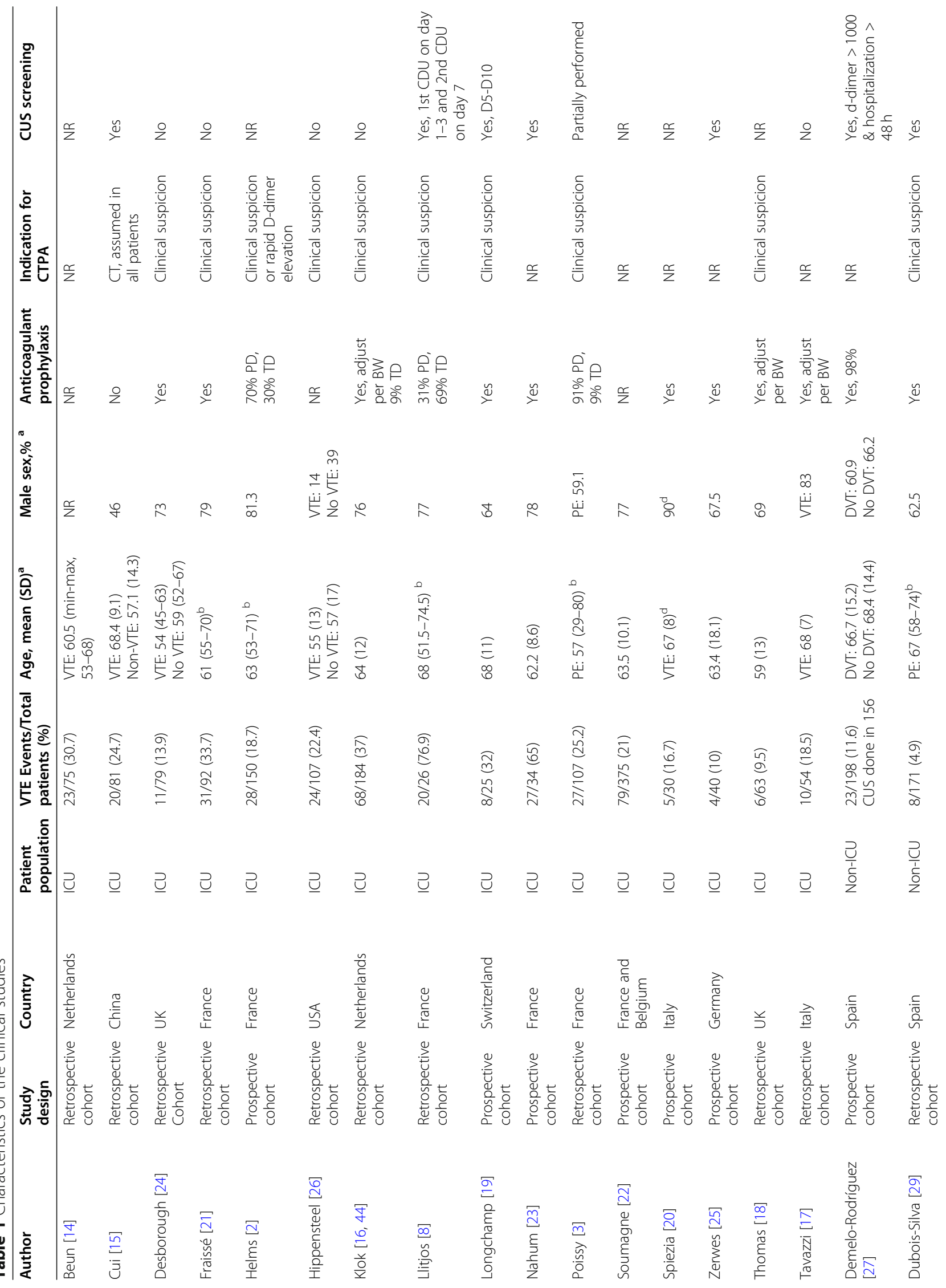




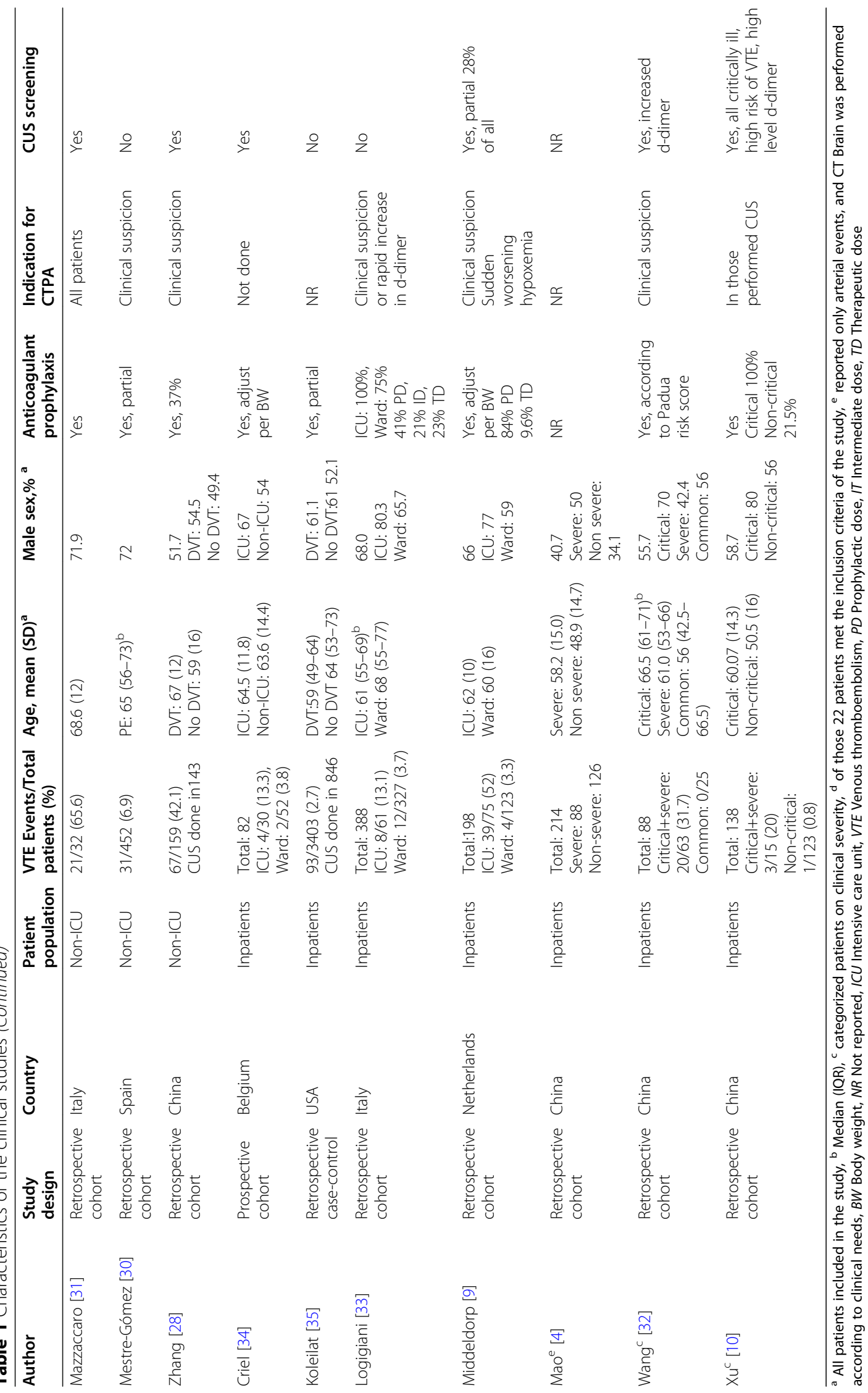




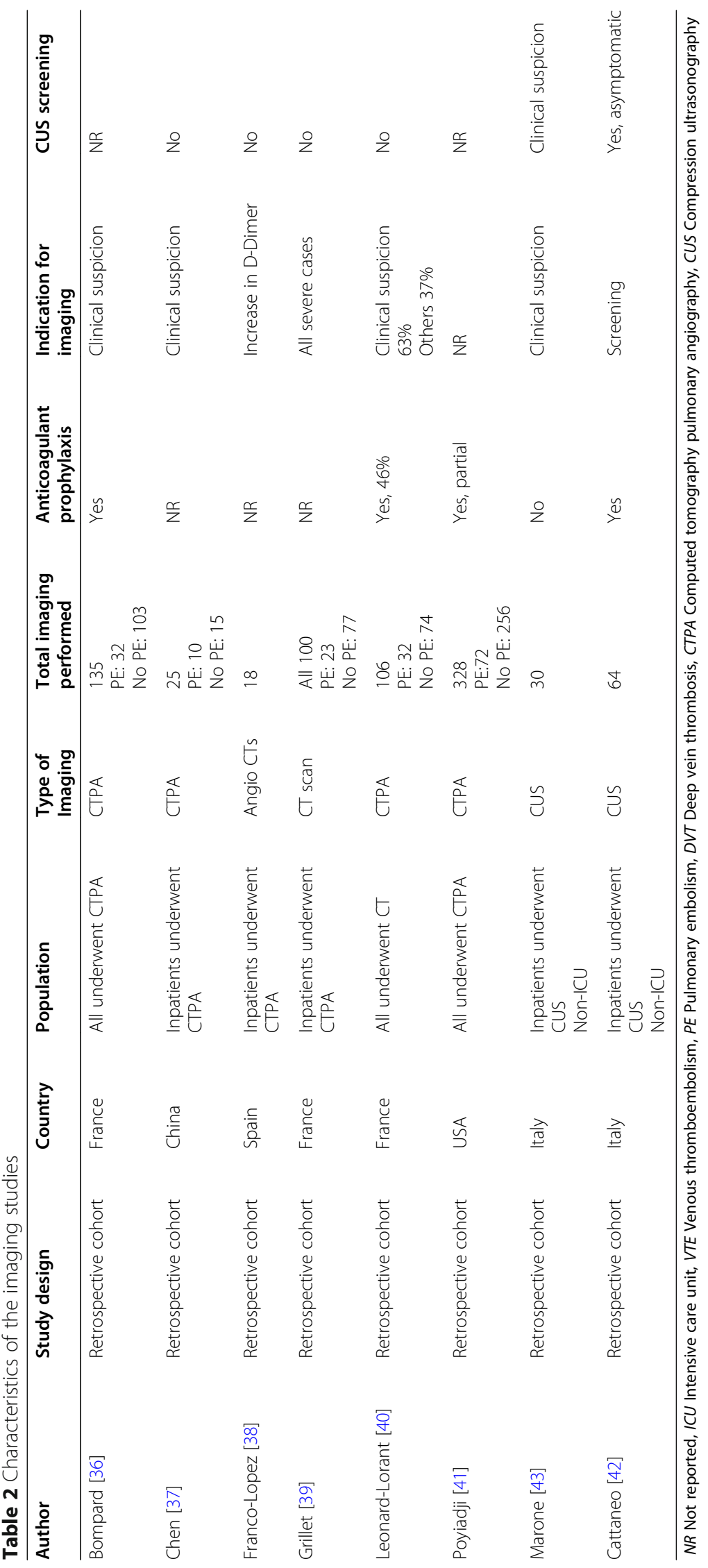




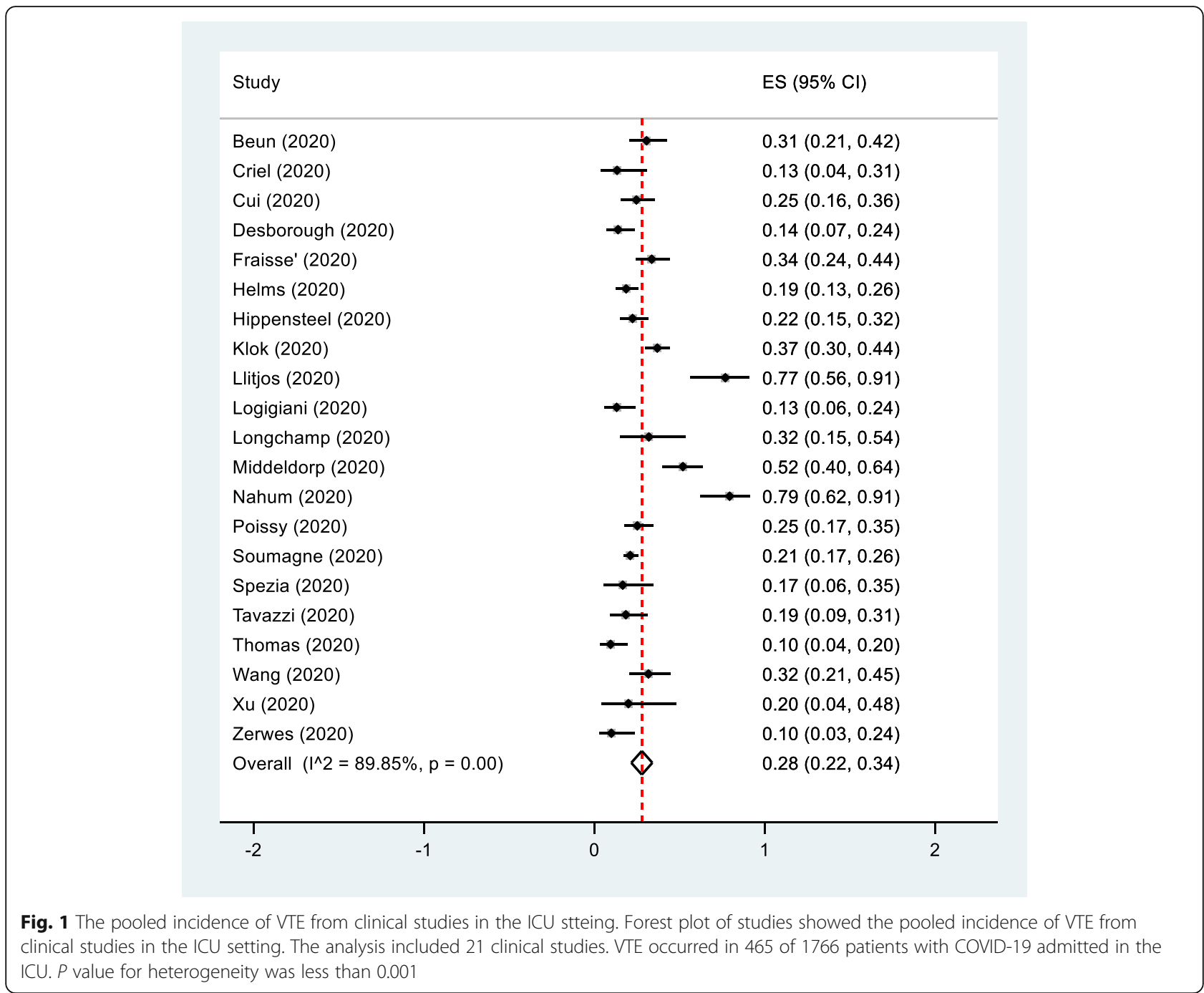

$40 \%$ (95\% CI, 29-50\%), 41\% (95\% CI, 26-56\%), 27\% (95\% CI, 20-34\%), 16\% (95\% CI, 10-21\%), and 12\% (95\% CI, 6-17\%), respectively. Each study from Switzerland, USA, Belgium and Germany demonstrated the VTE incidence of $32 \%$ (95\% CI, 15-54\%), 22\% (95\% CI, 15-32\%), $13 \%$ (95\% CI, 4-31\%), and 10\% (95\% CI 3-24\%), respectively (Fig. 3).

VTE in non-ICU setting A total of 10 clinical studies reported VTE events in non-ICU setting were included in the analysis. In 171 of 1662 patients with COVID-19 admitted in the general ward, the pooled incidence of total VTE was 10\% (95\% CI, 6-14\%, $\left.\mathrm{I}^{2}=96.8\right)$. The pooled incidence of PE was $0 \%$. The pooled incidence of DVT was $1 \%\left(95 \% \mathrm{CI}, 1-2 \%, \mathrm{I}^{2}=96\right)$ (Figures S6-S8 in the supplementary index).

Subgroup analysis of VTE based on anticoagulant prophylaxis was not performed since all had anticoagulant prophylaxis. There was no significant interaction on subgroup analysis based on eastern or western countries. Subgroup analyses based on CUS screening revealed a significant interaction between subgroups $(p=0.007)$. In 8 studies of the CUS screening subgroup, the incidence of VTE was $12 \%$ (95\% CI, 7-17\%). In 2 studies of the no CUS screening subgroup, the incidence of VTE was $5 \%$ (95\% CI, 2-6\%) (Figure S9-S10 in supplementary index).

Arterial thrombosis in the ICU setting A total of 7 clinical studies in the ICU setting reported on arterial thrombotic events, including myocardial infarction, ischemic stroke, and limb ischemia. Arterial thrombosis occurred in 30 of 713 patients with COVID-19 admitted in the ICU. The pooled incidence of total arterial thrombosis was 3\% (95\% CI, 2-5\%, $\mathrm{I}^{2}=4.1$ ) (Figure S11 in the supplementary index).

Arterial thrombosis in the non-ICU setting Two clinical studies reported on arterial thrombotic events 


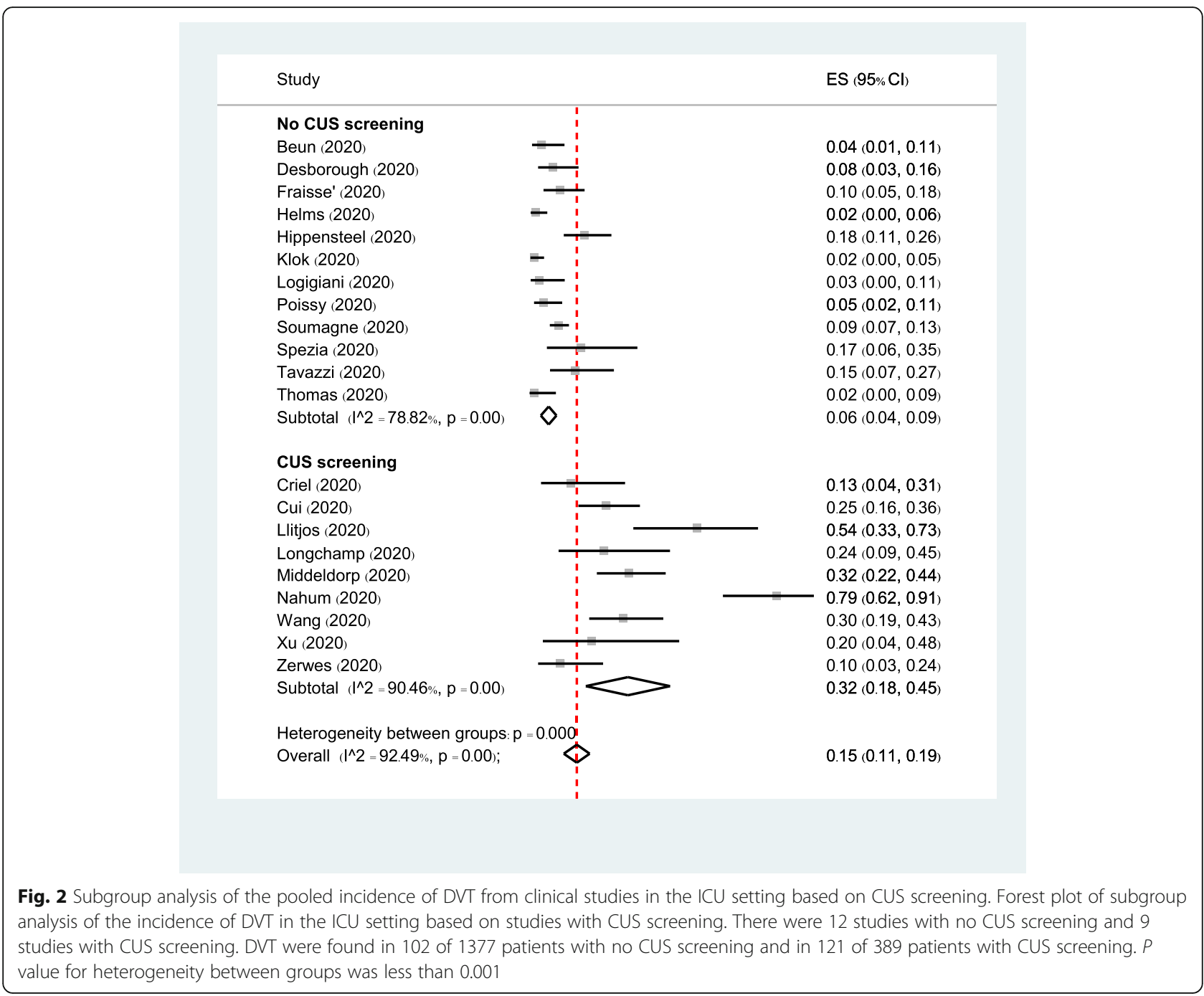

including ischemic stroke and myocardial infarction in the non-ICU setting. Arterial thrombosis occurred in 10 of 453 patients with COVID-19 admitted in the nonICU. The pooled incidence of total arterial thrombosis was $2 \%\left(95 \% \mathrm{CI}, 0-3 \%, \mathrm{I}^{2}=0\right)$ (Figure S12 in the supplementary index).

Mortality Six clinical studies reported the number of patients with VTE who died in the ICU setting. The overall mortality rate was $6 \%\left(3-10 \%, \mathrm{I}^{2}=63.8\right)$ (Figure S13 in the supplementary index).

\section{Imaging studies}

A total of 8 imaging studies were included. VTE was found in 261 of 949 imaging performed in patients with COVID-19 requiring hospitalization. The pooled incidence of total VTE was $29 \%$ (95\% CI, 15-42\%, I $\left.{ }^{2}=97.5\right)$ (Figure S14 in the supplementary index). Since each imaging study focused and reported on a specific type of imaging, we analyzed separately for imaging studies focusing on either CTPA or CUS study. In six imaging studies focusing on CTPA, the pooled incidence of PE was $26 \%\left(95 \% \mathrm{CI}, 21-31 \%, \mathrm{I}^{2}=40.8\right)$. In 2 imaging studies focusing on CUS, the pooled incidence of DVT was 0\% (Figure S15-16 in supplementary index).

For the location of PE reported on imaging studies focusing on CTPA, one study did not report the location of thrombus, and one study reported sites of thrombus of subsegmental, segmental, and lobar artery together. Four imaging studies focusing on CTPA reported the location of thrombus by distal (subsegmental, segmental artery) vs. proximal (lobar and more proximal part) artery. Of $144 \mathrm{PE}$ detected, distal PE was found in 81 (56\%), and proximal PE was found in 36 (35\%).

\section{Discussion}

Since evidence of an increased risk of thromboembolism in hospitalized COVID-19 patients emerged, several 


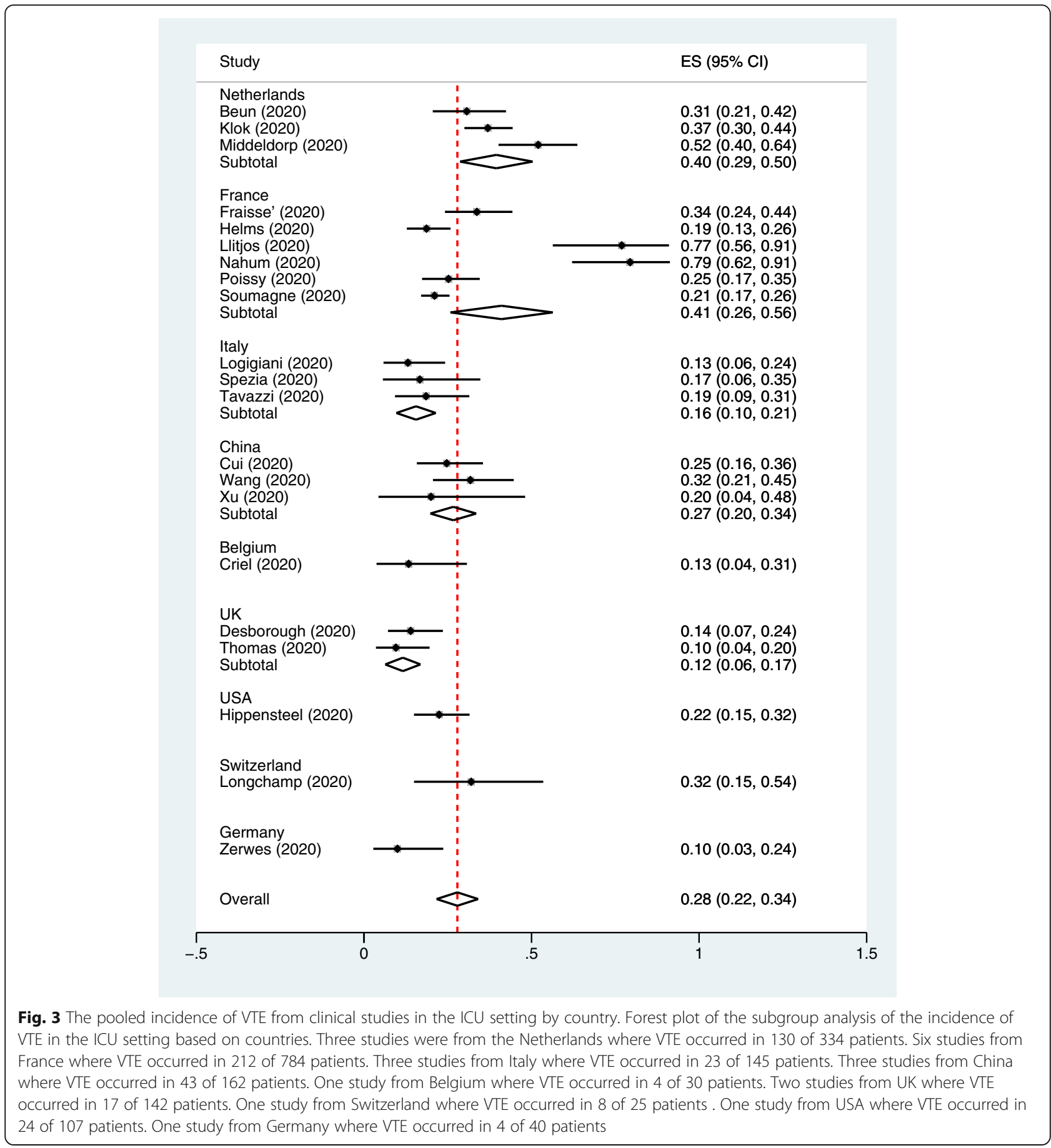

observational studies have reported specific outcomes on venous or arterial thrombosis. We systematically searched and illustrated the pooled incidence of venous and arterial thromboembolism in various clinical settings. We found that in patients requiring ICU admission had a higher incidence of VTE (28\%) than those in a non-ICU setting (10\%). Several studies demonstrated a significant increase in D-dimer and fibrinogen levels, which reflected the hypercoagulability state in COVID-19 ICU patients [20,45].
It is hypothesized that in severe clinical COVID-19 pneumonia, the massive release of inflammatory mediators caused by viral replication might be contributed to endothelial injury and intravascular thrombosis [46]. Our findings support the association between clinical severity and hypercoagulable state causing by COVID- 19 .

In the ICU setting, the pooled incidence of VTE was $28 \%$ with high heterogeneity. Prespecified subgroup analyses, including anticoagulant prophylaxis, provided similar results 
as the primary analysis. The interpretation of this subgroup analysis requires caution since only 4 studies did not utilize anticoagulant prophylaxis. In addition, among studies that utilize anticoagulant prophylaxis, criteria for anticoagulant prophylaxis and the dosage are varied. However, our data demonstrated that breakthrough VTE on anticoagulant prophylaxis occurred in approximately $29 \%$ in the ICU setting. In patients with COVID-19 with severe clinical severity or required ICU admission, prophylactic-intensity anticoagulation might not be sufficient. Whether a higher intensity anticoagulant could effectively prevent the venous thrombotic events in critically ill patients with COVID-19 is unknown. Several randomized controlled trials looking at the appropriate intensity of LMWH prophylaxis are still ongoing.

Our finding of a high incidence of VTE in the ICU setting was likely driven by the incidence of DVT rather than PE. The pooled incidence of PE was lower than we expected. When we performed post hoc subgroup analysis based on countries, studies from the Netherland and France had a higher pooled incidence of PE ranged from 17 to $27 \%$, whereas the studies from Italy and UK reported the lower incidence of $\mathrm{PE}$, which ranged from 3 to $7 \%$. The significant between-country differences in incidence reflect more stringent diagnostic workup procedures in the countries with higher reported incidence. Other confounding factors also could contribute to this finding. The indications for CTPA were varied between studies. Most studies performed CTPA based on clinical suspicion, whereas some studies based on a high Ddimer level or only in patients with DVT. Several studies did not mention performing CTPA or the indication for CTPA, which could underestimate the incidence of PE.

In China, the incidence of VTE in the ICU setting was $26 \%$. Here in our academic center in Thailand, we also found a significant number of PE in severe COVID-19 pneumonia patients. There were 3 symptomatic PE out of 14 severe COVID-19 pneumonia requiring ICU admission. At the time of events, all three patients did not receive anticoagulant prophylaxis [47]. Therefore, the incidence of VTE in severe COVID-19 requiring ICU admission in our center was $21.4 \%$. There was no VTE among 130 non-severe COVID-19. It is noted that we did not perform routine CUS screening in our patients.

The incidence of DVT in the ICU setting was significantly higher in studies that performed CUS screening than those studies which did not $(32 \%$ vs. $6 \%$, respectively). Though this finding was as expected since the more imaging performed, the more DVT events detected. The significance of asymptomatic DVT in the ICU setting detected by CUS is still debating. Whether a high incidence of DVT detected on CUS has an impact on the development of PE or mortality was unknown. Given the high risk of transmission of SARS-CoV-2 to health care personnel, recent expert guidance suggests against routine CUS screening in patients with COVID19 requiring ICU admission [48].

The incidence of VTE in COVID-19 in the non-ICU setting was $10 \%$. Subgroup analysis based on CUS screening revealed significant interaction. However, the credibility of the subgroup analysis was low. Most VTE incidences were driven by DVT dominating by Zhang et al. study (DVT incidence $=42 \%$ ) and by PE dominating by Mazzaccaro et al. study (PE incidence $=72 \%$, respectively) [28, 31]. In Zhang study, anticoagulant prophylaxis was given in $37 \%$ of patients whereas in other studies anticoagulant prophylaxis was utilized in more than $80 \%$ of patients. In another study from China by $\mathrm{Xu}$ and coworkers [10], anticoagulant prophylaxis was also partially given in $22 \%$ of patients, but the incidence of DVT was quite low (1\%). This could be explained by a difference in the baseline risk of VTE for which the proportion of patients with high-risk VTE (Padua score $\geq 4$ ) was much higher in the study by Zhang than those in the study by Xu et al. (65\% vs. $7 \%$ ). A high incidence of PE in a study by Mazzaccaro could be due to CTPA screening for PE in all patients rather than in those with clinical suspicion. Overall, the incidence of VTE is low in the non-ICU setting. Anticoagulant prophylaxis should be considered in the non-ICU setting, especially in patients with high risk for VTE.

Among the imaging studies which reported PE events detected by CTPA or DVT detected by CUS, the incidences of PE and DVT were 26 and 33\%, respectively. Most studies selected patients who underwent CTPA or CUS regardless of clinical severity status. Thus, the interpretation was limited. Findings of imaging on CTPA demonstrated that thrombus was more commonly occurred in the distal part (subsegmental and segmental artery) rather than a more proximal pulmonary artery. This could reflect the microvascular thrombosis "in situ" caused by endothelial injury and local inflammation $[46,49]$.

Data on VTE-related death were limited. Few studies reported the outcome of death in patients with VTE, but it could not be assumed to be VTE-related death in all patients. Many clinical and laboratory factors, including older age, sex, clinical severity assessed by using SOFA score, and high D-dimer were associated with mortality $[50,51]$.

Few arterial thrombotic events have been reported. Most events were ischemic stroke, and few were myocardial infarction. Though several case reports and case series demonstrated the possibly increased risk of arterial thrombosis, we did not include those studies in the analysis since there was no data available for incidence calculation, and the study designs are subjected to selection bias. 
Our study has several strength. We performed a systematic literature search, including grey literature with no language restriction. Full-text eligibility and risk of bias were reviewed by two independent researchers. However, there are some limitations. Most studies were retrospective in design with a small sample size. Thus they were subjected to risk of selection bias. High heterogeneity between groups was presented in most analyses, although $\mathrm{I}^{2}$ is possibly not a reliable indicator of true heterogeneity among prevalence meta-analyses [52]. One post hoc subgroup analysis was able to demonstrate the subgroup effect but prespecified subgroup analyses did not reveal significant interaction. CTPA for PE detection was not routinely performed in all patients in the ICU. Hence, the true incidence of PE could be underestimated. However, most studies performed CTPA based on clinical suspicion, which represented the clinically significant PE.

\section{Conclusion}

The incidence of VTE was 28 and 10\% in COVID-19 patients in the ICU and non-ICU settings, respectively. The incidence of DVT was significantly higher in studies that incorporated the CUS screening protocol. The incidence of ATE in the ICU setting was low. VTE prophylactic measures should be given to all hospitalized patients with COVID-19, especially in the ICU setting. Since approximately one-fourth of patients admitted to the ICU setting developed VTE, careful monitoring of the patients for VTE and its complications is strongly advised. The optimization of anticoagulant dosing to prevent VTE is currently under investigation.

\section{Supplementary Information}

The online version contains supplementary material available at https://doi. org/10.1186/s12959-020-00248-5.

\section{Additional file 1}

\section{Abbreviations}

COVID-19: Coronavirus disease 2019; VTE: Venous thromboembolism; PE: Pulmonary embolism; DVT: Deep vein thrombosis; ATE: Arterial thromboembolism; Cl: Confidence interval; ICU: Intensive care unit; CUS: Compression ultrasound; CTPA: Computed tomography pulmonary angiography; UK: United Kingdom; USA: United States of America; SARS-CoV2: Severe acute respiratory syndrome coronavirus 2; RT-PCR: Real-time polymerase chain reaction

\section{Acknowledgements}

Not applicable.

\section{Authors' contributions}

K. B. and P. Numthavej designed the methods; K. B. and P.C. performed study selection; K.B. and P. Numthavej performed data extraction, study quality assessment, and analysis; K. B. wrote the manuscript; and N.N., S.P., A.P., P. Niparuck, and P. Angchaisuksiri critically revised the manuscript. The author(s) read and approved the final manuscript.
Funding

Not applicable.

Availability of data and materials

Not applicable.

Ethics approval and consent to participate

Not applicable.

Consent for publication

Not applicable.

\section{Competing interests}

The authors declare no competing financial interests.

\section{Author details}

${ }^{1}$ Department of Medicine, Faculty of Medicine Ramathibodi Hospital, Mahidol University, Bangkok, Thailand. ${ }^{2}$ Department of Clinical Epidemiology and Biostatistics, Faculty of Medicine Ramathibodi Hospital, Mahidol University, Bangkok, Thailand. ${ }^{3}$ Chakri Naruebodindra Medical Institute, Faculty of Medicine Ramathibodi Hospital, Mahidol University, Samut Prakan, Thailand.

Received: 21 August 2020 Accepted: 10 November 2020 Published online: 23 November 2020

\section{References}

1. Connors JM, Levy JH. COVID-19 and its implications for thrombosis and anticoagulation. Blood. 2020;135(23):2033-40.

2. Helms J, Tacquard C, Severac F, Leonard-Lorant I, Ohana M, Delabranche $X$, et al. High risk of thrombosis in patients with severe SARS-CoV-2 infection: a multicenter prospective cohort study. Intensive Care Med. 2020;46(6):1089-98.

3. Poissy J, Goutay J, Caplan M, Parmentier E, Duburcq T, Lassalle F, et al. Pulmonary embolism in patients with COVID-19: awareness of an increased prevalence. Circulation. 2020:142(2):184-6.

4. Mao L, Jin H, Wang M, Hu Y, Chen S, He Q, et al. Neurologic manifestations of hospitalized patients with coronavirus disease 2019 in Wuhan, China. JAMA Neurol. 2020;10:10

5. Kashi M, Jacquin A, Dakhil B, Zaimi R, Mahe E, Tella E, et al. Severe arterial thrombosis associated with Covid-19 infection. Thromb Res. 2020;192:75-7.

6. Bellosta R, Luzzani L, Natalini G, Pegorer MA, Attisani L, Cossu LG, et al. Acute limb ischemia in patients with COVID-19 pneumonia. J Vasc Surg Venous Lymphat Disord. 2020;S0741-5214(20):31080-6.

7. Oxley TJ, Mocco J, Majidi S, Kellner CP, Shoirah H, Singh IP, et al. Largevessel stroke as a presenting feature of Covid-19 in the young. N Engl J Med. 2020:382(20):e60

8. Llitjos JF, Leclerc M, Chochois C, Monsallier JM, Ramakers M, Auvray M, et al. High incidence of venous thromboembolic events in anticoagulated severe COVID-19 patients. J Thromb Haemost. 2020;18(7):1743-6.

9. Middeldorp S, Coppens M, van Haaps TF, Foppen M, Vlaar AP, Muller MCA, et al. Incidence of venous thromboembolism in hospitalized patients with COVID-19. J Thromb Haemost. 2020;05:05.

10. Jin-fu Xu, Lan Wang, Lan Zhao et al. Risk assessment of venous thromboembolism and bleeding in COVID-19 patients, 24 March 2020, PREPRINT (Version 1) available at Research Square [+https://doi.org/10. 21203/rs.3.rs-18340/v1+].

11. Migliavaca CB, Stein C, Colpani V, Munn Z, Falavigna M; Prevalence Estimates Reviews - Systematic Review Methodology Group (PERSyst). Quality assessment of prevalence studies: a systematic review. J Clin Epidemiol. 2020;127:59-68. https://doi.org/10.1016/j.jclinepi.2020.06.039. Epub ahead of print. PMID: 32679313.

12. Hoy D, Brooks P, Woolf A, Blyth F, March L, Bain C, et al. Assessing risk of bias in prevalence studies: modification of an existing tool and evidence of interrater agreement. J Clin Epidemiol. 2012:65(9):934-9.

13. Nyaga VN, Arbyn M, Aerts M. Metaprop: a Stata command to perform metaanalysis of binomial data. Arch Public Health. 2014;72(1):39.

14. Beun R, Kusadasi N, Sikma M, Westerink J, Huisman A. Thromboembolic events and apparent heparin resistance in patients infected with SARS-CoV-2. Int J Lab Hematol. 2020:42(Suppl 1):19-20. 
15. Cui S, Chen S, Li X, Liu S, Wang F. Prevalence of venous thromboembolism in patients with severe novel coronavirus pneumonia. J Thromb Haemost. 2020;18(6):1421-4.

16. Klok FA, Kruip M, van der Meer NJM, Arbous MS, Gommers D, Kant KM, et al. Confirmation of the high cumulative incidence of thrombotic complications in critically ill ICU patients with COVID-19: an updated analysis. Thromb Res. 2020;191:148-50.

17. Tavazzi G, Civardi L, Caneva L, Mongodi S, Mojoli F. Thrombotic events in SARS-CoV-2 patients: an urgent call for ultrasound screening. Intensive Care Med. 2020;46(6):1121-3.

18. Thomas W, Varley J, Johnston A, Symington E, Robinson M, Sheares K, et al. Thrombotic complications of patients admitted to intensive care with COVID-19 at a teaching hospital in the United Kingdom. Thromb Res. 2020; 191:76-7.

19. Longchamp A, Longchamp J, Manzocchi-Besson S, Whiting L, Haller C, Jeanneret $S$, et al. Venous thromboembolism in critically ill patients with COVID-19: results of a screening study for deep vein thrombosis. Res Pract Thromb Haemost. 2020;4(5):842-7.

20. Spiezia L, Boscolo A, Poletto F, Cerruti L, Tiberio I, Campello E, et al. COVID 19-related severe hypercoagulability in patients admitted to intensive care unit for acute respiratory failure. Thromb Haemost. 2020;120(6):998-1000

21. Fraisse M, Logre E, Pajot O, Mentec H, Plantefeve G, Contou D. Thrombotic and hemorrhagic events in critically ill COVID-19 patients: a French monocenter retrospective study. Crit Care. 2020;24(1):275.

22. Soumagne T, Lascarrou JB, Hraiech S, Horlait G, Higny J, d'Hondt A, et al. Factors associated with pulmonary embolism among coronavirus disease 2019 acute respiratory distress syndrome: a multicenter study among 375 patients. Crit Care Explor. 2020;2(7):e0166.

23. Nahum J, Morichau-Beauchant T, Daviaud F, Echegut P, Fichet J, Maillet JM, et al. Venous thrombosis among critically ill patients with coronavirus disease 2019 (COVID-19). JAMA Netw Open. 2020;3(5):e2010478.

24. Desborough MJR, Doyle AJ, Griffiths A, Retter A, Breen KA, Hunt BJ. Imageproven thromboembolism in patients with severe COVID-19 in a tertiary critical care unit in the United Kingdom. Thromb Res. 2020;193:1-4.

25. Zerwes S, Hernandez Cancino F, Liebetrau D, Gosslau Y, Warm T, Markl B, et al. Increased risk of deep vein thrombosis in intensive care unit patients with CoViD-19 infections?-preliminary data. Chirurg. 2020;91(7):588-94.

26. Hippensteel JA, Burnham EL, Jolley SE. Prevalence of venous thromboembolism in critically ill patients with COVID-19. Br J Haematol. 2020;190(3):e134-e7.

27. Demelo-Rodriguez P, Cervilla-Munoz E, Ordieres-Ortega L, Parra-Virto A, Toledano-Macias M, Toledo-Samaniego N, et al. Incidence of asymptomatic deep vein thrombosis in patients with COVID-19 pneumonia and elevated D-dimer levels. Thromb Res. 2020;192:23-6.

28. Zhang L, Feng X, Zhang D, Jiang C, Mei H, Wang J, et al. Deep vein thrombosis in hospitalized patients with COVID-19 in Wuhan, China: prevalence, risk factors, and outcome. Circulation. 2020;142(2):114-28.

29. Dubois-Silva Á, Barbagelata-López C, Mena Á, Piñeiro-Parga P, Llinares-García D, Freire-Castro S. Pulmonary embolism and screening for concomitant proximal deep vein thrombosis in noncritically ill hospitalized patients with coronavirus disease 2019. Intern Emerg Med. 2020;15(5):865-70.

30. Mestre-Gomez B, Lorente-Ramos RM, Rogado J, Franco-Moreno A, Obispo B, Salazar-Chiriboga D, et al. Incidence of pulmonary embolism in non-critically ill COVID-19 patients. Predicting factors for a challenging diagnosis. J Thromb Thrombolysis. 2020:1-7. Advance online publication. https://doi.org/10.1007/s11239-020-02190-9.

31. Mazzaccaro D, Giacomazzi F, Giannetta M, Varriale A, Scaramuzzo R, Modafferi A, et al. Non-Overt Coagulopathy in Non-ICU Patients with Mild to Moderate COVID-19 Pneumonia. J Clin Med. 2020;9(6):1781.

32. Wang W, Sun $Q$, Bao Y, Liang M, Meng Q, Chen $H$, et al. Analysis of risk factors for the thromboembolic events from 88 patients with COVID-19 pneumonia in Wuhan. China: A Retrospective Report; 2020.

33. Lodigiani C, lapichino G, Carenzo L, Cecconi M, Ferrazzi P, Sebastian T, et al. Venous and arterial thromboembolic complications in COVID-19 patients admitted to an academic hospital in Milan. Italy Thromb Res. 2020;191:9-14.

34. Criel $M$, Falter $M$, Jaeken J, Van Kerrebroeck $M$, Lefere I, Meylaerts $L$, et al. Venous thromboembolism in SARS-CoV-2 patients: only a problem in ventilated ICU patients, or is there more to it? Eur Respir J. 2020:56(1): 2001201. https://doi.org/10.1183/13993003.01201-2020.

35. Koleilat I, Galen B, Choinski K, Hatch AN, Jones DB, Billett H, et al. Clinical characteristics of acute lower extremity deep venous thrombosis diagnosed by duplex in patients hospitalized for coronavirus disease 2019. J Vasc Surg Venous Lymphat Disord:2020. S2213-333X(20)30348-6. Advance online publication. https://doi.org/10.1016/j.jvsv.2020.06.012.

36. Bompard F, Monnier $H$, Saab I, Tordjman M, Abdoul $H$, Fournier $L$, et al. Pulmonary embolism in patients with Covid-19 pneumonia. Eur Respir J. 2020;56(1):2001365. https://doi.org/10.1183/13993003.01365-2020.

37. Chen J, Wang X, Zhang S, Liu B, Wu X, Wang Y, Wang X, Yang M, Sun J, Xie $Y$. Findings of acute pulmonary embolism in COVID-19 patients. Available at SSRN 3548771. 2020 Mar 1.

38. Franco-López AEPJ, Vicente GN. Tromboembolismo Pulmonar en los pacientes con COVID-19. Angiografía con tomografía computarizada: resultados preliminares. JONNPR. 2020;5(6):616-30.

39. Grillet F, Behr J, Calame P, Aubry S, Delabrousse E. Acute Pulmonary Embolism Associated with COVID-19 Pneumonia Detected with Pulmonary CT Angiography. Radiology. 2020;296(3):E186-8.

40. Leonard-Lorant I, Delabranche X, Severac F, Helms J, Pauzet C, Collange O, et al. Acute Pulmonary Embolism in Patients with COVID-19 at CT Angiography and Relationship to d-Dimer Levels. Radiology. 2020;296(3): E189-91.

41. Poyiadji N, Cormier P, Patel PY, Hadied MO, Bhargava P, Khanna K, et al. Acute pulmonary embolism and COVID-19. Radiology. 2020:201955. Advance online publication. https://doi.org/10.1148/radiol.2020201955.

42. Cattaneo M, Bertinato EM, Birocchi S, Brizio C, Malavolta D, Manzoni M, et al. Pulmonary embolism or pulmonary thrombosis in COVID-19? Is the recommendation to use high-dose heparin for Thromboprophylaxis justified? Thromb Haemost. 2020;29:29.

43. Marone EM, Rinaldi LF. Upsurge of deep venous thrombosis in patients affected by COVID-19: preliminary data and possible explanations. J Vasc Surg Venous Lymphat Disord. 2020;8(4):694-5.

44. Klok FA, Kruip M, van der Meer NJM, Arbous MS, Gommers D, Kant KM, et al. Incidence of thrombotic complications in critically ill ICU patients with COVID-19. Thromb Res. 2020;191:145-7.

45. Panigada M, Bottino N, Tagliabue P, Grasselli G, Novembrino C, Chantarangkul V, et al. Hypercoagulability of COVID-19 patients in intensive care unit: a report of thromboelastography findings and other parameters of hemostasis. J Thromb Haemost. 2020;18(7):1738-42.

46. Price LC, McCabe C, Garfield B, Wort SJ. Thrombosis and COVID-19 pneumonia: the clot thickens! Eur Respir J. 2020;56(1):2001608.

47. Nanthatanti N, Phusanti S, Chantrathammachart P, Thammavaranucupt K, Angchaisuksiri P, Sungkanuparph S. Left ventricular thrombus and pulmonary embolism: A case series of thrombosis in COVID-19 in Thai patients. Res Pract Thromb Haemost. 2020;4(7):1224-9.

48. Moores LK, Tritschler T, Brosnahan S, Carrier M, Collen JF, Doerschug K, et al. Prevention, diagnosis, and treatment of VTE in patients with COVID-19: CHEST guideline and expert panel report. Chest. 2020;158(3):1143-63.

49. Varga Z, Flammer AJ, Steiger $P$, Haberecker M, Andermatt R, Zinkernagel AS, et al. Endothelial cell infection and endotheliitis in COVID-19. Lancet. 2020; 395(10234):1417-8.

50. Zhang J, Wang X, Jia X, Li J, Hu K, Chen G, et al. Risk factors for disease severity, unimprovement, and mortality in COVID-19 patients in Wuhan, China. Clin Microbiol Infect. 2020;26(6):767-72

51. Yang $K$, Sheng Y, Huang C, Jin Y, Xiong N, Jiang K, et al. Clinical characteristics, outcomes, and risk factors for mortality in patients with cancer and COVID-19 in Hubei, China: a multicentre, retrospective, cohort study. Lancet Oncol. 2020;21(7):904-13.

52. Borges Migliavaca C, Stein C, Colpani V, Barker TH, Munn Z, Falavigna M, et al. How are systematic reviews of prevalence conducted? A methodological study. BMC Med Res Methodol. 2020;20(1):96.

\section{Publisher's Note}

Springer Nature remains neutral with regard to jurisdictional claims in published maps and institutional affiliations. 